MATHEMATICS OF COMPUTATION

Volume 69, Number 230, Pages 797-810

S 0025-5718(99)01143-6

Article electronically published on August 18, 1999

\title{
TABLES OF CURVES WITH MANY POINTS
}

\author{
GERARD VAN DER GEER AND MARCEL VAN DER VLUGT
}

\begin{abstract}
These tables record results on curves with many points over finite fields. For relatively small genus $(0 \leq g \leq 50)$ and $q$ a small power of 2 or 3 we give in two tables the best presently known bounds for $N_{q}(g)$, the maximum number of rational points on a smooth absolutely irreducible projective curve of genus $g$ over a field $\mathbb{F}_{q}$ of cardinality $q$. In additional tables we list for a given pair $(g, q)$ the type of construction of the best curve so far, and we give a reference to the literature where such a curve can be found.
\end{abstract}

\section{INTRODUCTION}

In recent years the question of how many points a curve of genus $g$ over a finite field $\mathbb{F}_{q}$ can have has attracted a lot of attention. This was motivated partly by possible applications in coding theory and cryptography, but also by the fact that the question represents an attractive mathematical challenge.

It is well known that a smooth absolutely irreducible projective curve of genus $g$ over a finite field $\mathbb{F}_{q}$ can possess at most $q+1+2 g \sqrt{q}$ rational points. By a curve we shall mean in this paper a smooth absolutely irreducible projective curve defined over a finite field. The bound mentioned is the celebrated Hasse-Weil bound, proved by Hasse for $g=1$ and by Weil in general. We denote by $N_{q}(g)$ the maximum number of rational points on a curve of genus $g$ over $\mathbb{F}_{q}$. The Hasse-Weil bound implies

$$
N_{q}(g) \leq q+1+[2 g \sqrt{q}]
$$

where $[x]$ is the integer part of $x \in \mathbb{R}$.

After Weil proved his bound around 1940, the question of how many rational points may lie on a curve over a finite field $\mathbb{F}_{q}$ remained untouched for many years. In 1980 Goppa came up with the beautiful idea of associating an error-correcting code to a linear system on a curve over a finite field, see $[G]$. In order to construct good codes one needs curves with many points, and thus Goppa's work led to a revival of interest in rational points on curves over finite fields. Applications in cryptography and recent constructions of quasi-random point sets also require curves with many points, and added a further impetus to work in the field.

In 1981 Ihara showed in [I] by a simple and elegant argument that

$$
N_{q}(g) \leq q+1+\left[\left(\sqrt{(8 q+1) g^{2}+4\left(q^{2}-q\right) g}-g\right) / 2\right] .
$$

Received by the editor October 2, 1997 and, in revised form, April 28, 1998.

1991 Mathematics Subject Classification. Primary 11G20, 14G15; Secondary 14H05.

(C)2000 American Mathematical Society 
For $g>(q-\sqrt{q}) / 2$ this bound is better than Weil's bound and gives the asymptotic bound

$$
A(q):=\limsup _{g \rightarrow \infty} \frac{N_{q}(g)}{g} \leq \sqrt{2 q+\frac{1}{4}}-\frac{1}{2} .
$$

Ihara also showed that if $q$ is a square one has $A(q) \geq \sqrt{q}-1$, using a sequence of modular curves. Refining Ihara's idea to derive (1), Drinfeld and Vladut proved that

$$
A(q) \leq \sqrt{q}-1 .
$$

In [S1] Serre started the investigation of the actual value of $N_{q}(g)$. One has $N_{q}(0)=q+1$. For $g=1,2$ there are explicit formulas for $N_{q}(g)$. From [S2], [S4] we quote the following result:

Proposition 1. Let $q=p^{m}$ and set $\mu=[2 \sqrt{q}]$. For $g=1$ one has $N_{q}(1)=$ $q+1+\mu$, except when $m$ is odd, $m \geq 3$ and $p$ divides $\mu$, in which case we have $N_{q}(1)=q+\mu$. Similarly, for $g=2$ we have $N_{q}(2)=q+1+2 \mu$ except in the following cases:

i) $N_{4}(2)=10, N_{9}(2)=20$;

ii) $m$ odd, $p$ divides $\mu$;

iii) $m$ odd and $q$ of the form $x^{2}+1, x^{2}+x+1$ or $x^{2}+x+2$ for $x \in \mathbb{Z}$.

In cases ii) and iii) we have $N_{q}(2)=q+2 \mu$ if $2 \sqrt{q}-\mu>(\sqrt{5}-1) / 2$, or $N_{q}(2)=q+2 \mu-1$ else.

In [S1] Serre used a little arithmetic to show that the Hasse-Weil bound may be sharpened to

$$
N_{q}(g) \leq q+1+g[2 \sqrt{q}] .
$$

In the same paper Serre introduced the idea of using a 'formule explicite' in analogy with number theory for obtaining a better upper bound for $N_{q}(g)$. Oesterlé used methods from linear programming to perfect this idea, see [S4].

In the tables we shall use as upper bound for $N_{q}(g)$ the best bound that these estimates of Hasse-Weil, Ihara, Serre and Oesterlé provide. We also take into account slight improvements by 1,2 , or 3 of these upper bounds. They result from the following facts.

Proposition $2([\mathrm{~F}-\mathrm{T}])$. If $q$ is a square and if $C$ is a curve of genus $g$ which attains the Hasse-Weil bound, then

$$
g \leq(\sqrt{q}-1)^{2} / 4 \quad \text { or } \quad g=(q-\sqrt{q}) / 2 .
$$

Proposition 3 ([S4]). A curve of genus $\geq 3$ with $\# C\left(\mathbb{F}_{q}\right)<q+1+g[2 \sqrt{q}]$ satisfies $\# C\left(\mathbb{F}_{q}\right) \leq q-1+g[2 \sqrt{q}]$.

Proposition 4. One has the following explicit results:

1) $N_{2}(7)=10$;

2) $N_{3}(5) \leq 13, N_{3}(7)=16, N_{8}(6) \leq 35$, and $N_{9}(5) \leq 35$;

3) $N_{4}(4)=15$ and $N_{9}(4)=30$;

4) $N_{27}(3)=56$.

Here 1) and 2) are obtained by an analysis of the Frobenius eigenvalues and are due to Serre [S4] and Lauter [L2], [L3] respectively. Result 3) was proved by Serre for $q=4$ and follows from $[\mathrm{S}-\mathrm{V}]$ for $q=9$. Also 4$)$ is due to Serre. Each of these improvements involves detailed considerations. 
Proposition 5 ([L3]). 1) For pairs $q=8, g \geq 4$ and $q \in\{27,32\}, g \geq 3$ we have $N_{q}(g) \leq q-1+g[2 \sqrt{q}]$. 2) For $q=2^{m}$ with even $m \geq 4$ and $(\sqrt{q}-1)^{2} / 4<g<$ $(q-\sqrt{q}) / 2$ we have $N_{q}(g) \leq q-2+2 g \sqrt{q}$.

Though it seems very difficult to improve the upper bounds for $N_{q}(g)$, one cannot expect in general that $N_{q}(g)$ equals the upper bound that we have, as examples over $\mathbb{F}_{2}$ and $\mathbb{F}_{3}$ already show. Therefore, to test how good these bounds really are, one tries to come as close to these bounds as one can by constructing curves with as many points as possible. With an eye towards feasibility of applications, it is important to have such curves in a form as explicit as possible.

The methods used for the construction of curves with many points are rather diverse, but roughly speaking one can distinguish the following approaches:

I Methods from general class field theory;

II Methods from class field theory based on Drinfeld modules of rank 1;

III Fibre products of Artin-Schreier curves;

IV Towers of curves with many points;

$\mathrm{V}$ Miscellaneous methods such as:

1) formulas for $N_{q}(1)$ and $N_{q}(2)$;

2) explicit curves, e.g. Hermitean curves, Klein's quartic, Artin-Schreier curves, Kummer extensions or curves obtained by computer search;

3 ) elliptic modular curves $X(n)$ associated to the full congruence subgroups $\Gamma(n)$

4) Deligne-Lusztig curves;

5) quotients of curves with many points.

Methods from general class field theory were used by Serre, Schoof, Lauter, Niederreiter and Xing, and Auer. They exploit subfields of Hilbert class fields or more generally of ray class fields of the function field of a given curve $C$ in which a substantial number of the rational points of $C$ split completely. General class field theory is a powerful weapon, but has the drawback that often it produces a mere existence result and not an explicit curve.

Constructing curves with many points by employing properties of Drinfeld modules of rank 1 was introduced by Niederreiter and Xing. When such a construction is applied to the case where the base curve $C$ is the projective line $\mathbb{P}^{1}$, one can produce good subfields of cyclotomic function fields which have the advantage of being explicit. For general base curves the curves produced correspond to subfields of narrow ray class fields, and explicit forms of these function fields are then much harder to find.

Fibre products are used by Stichtenoth, by van der Geer and van der Vlugt, and by Shabat. The method yields defining equations for the curves thus constructed. In category IV one finds mainly towers consisting of a combination of Kummer and Artin-Schreier extensions or composita of Kummer extensions. The function fields are explicit.

So far the curves constructed by method V-5 are all quotients of the Hermitean curve defined over $\mathbb{F}_{q^{2}}$ by

$$
x^{q+1}+y^{q+1}+z^{q+1}=0 .
$$




\section{THE TABLES}

For $g \leq 50$ and for $q=2^{m}$ with $1 \leq m \leq 7$ and $q=3^{m}$ with $1 \leq m \leq 4$ we present tables which list values of $N_{q}(g)$ or an interval in which $N_{q}(g)$ lies. Note that $g=50$ is the largest value for which the actual value $N_{2}(g)$ is known. We therefore restricted ourselves to $g \leq 50$. Of course $N_{q}(0)=q+1$ for all $q$, and it is omitted from the tables. If the precise value of $N_{q}(g)$ is not known, we give either an interval $[a, b]=\left[a_{q}(g), b_{q}(g)\right]$ or nothing. The meaning of the interval $[a, b]$ is: we know that there exists a curve with at least a rational points over $\mathbb{F}_{q}$, and the best upper bound by Hasse-Weil, Serre, Ihara, Oesterlé or other means says $N_{q}(g) \leq b$. In the lion's share of the cases the value of $a$ represents a curve with exactly a rational points; in about 20 cases (mostly constructed with method II), $a$ represents a lower bound for $N_{q}(g)$. Sometimes we entered no value. This happens if no curve with at least $[b / \sqrt{2}]$ rational points is known, i.e. if

$$
a_{q}(g)<\left[b_{q}(g) / \sqrt{2}\right] .
$$

The reason for this is that for $g \leq 50$ in many cases the upper bound $b_{q}(g)$ is Ihara's bound (1). Since the Drinfeld-Vladut asymptotic bound (3) is approximately $1 / \sqrt{2}$ times the asymptotic Ihara bound (2), we think it is reasonable to impose this qualification requirement for $g \leq 50$ to filter out curves which should be considered 'poor'.

Two main tables, 'Table $p=2$ ' and 'Table $p=3$ ', present values of the function $N_{q}(g)$ or an interval in which $N_{q}(g)$ lies. In additional tables $q=x$ : sources we list the construction method of a curve producing the value of $a_{q}(g)$ and the source where this curve occurs first.

Remarks. i) For $q=2$ one can find explicit curves realizing the lower bound for $g \in\{5,6,7,8,9,12,13,14,15\}$ in $[\mathrm{N}-\mathrm{X} 2]$, for $g=10$ in [G-V7] and for $g=11$ in [N-X1]. For $q=3,4, g=4$ there are explicit curves in [N-X3].

ii) A result communicated to us by R. Schoof (see [G-V4]) gives values for the lower bound $a_{q}(g)$ for the pairs $(q=2, g \in\{26,27,32,33,38,40,46,47,48\}),(q=$ $4, g \in\{6,16,44,45\})$ and $(q=8, g \in\{16,22,23,45\})$.

iii) The modular curves $X(9), X(11)$ and $X(13)$ yield the results for $(q=4, g \in$ $\{10,26,50\})$, and $X(8), X(10), X(11)$ and $X(13)$ yield the results for $(q=9, g \in$ $\{5,13,26,50\})$.

The results collected in our tables represent the work of many mathematicians. We tried to give credit to whom it is due, but may have failed due to ignorance. A closer look at the tables will convince the reader that there is still ample room for improvement. The tables should be seen as an attempt to record the state of the art. If the reader knows an improvement of an entry we shall appreciate if he/she let us know so that we can update or correct the tables. 
TABle $\mathrm{p}=2$

\begin{tabular}{|c|c|c|c|c|c|c|c|}
\hline$g \backslash q$ & 2 & 4 & 8 & 16 & 32 & 64 & 128 \\
\hline 1 & 5 & 9 & 14 & 25 & 44 & 81 & 150 \\
\hline 2 & 6 & 10 & 18 & 33 & 53 & 97 & 172 \\
\hline 3 & 7 & 14 & 24 & 38 & $63-64$ & 113 & 191-195 \\
\hline 4 & 8 & 15 & $25-27$ & $45-46$ & $70-75$ & 129 & $200-217$ \\
\hline 5 & 9 & $17-18$ & $29-32$ & $49-54$ & $76-86$ & $130-145$ & $227-239$ \\
\hline 6 & 10 & 20 & $33-35$ & 65 & 86-97 & 161 & $225-261$ \\
\hline 7 & 10 & $21-22$ & $33-39$ & $63-70$ & 90-108 & 177 & $258-283$ \\
\hline 8 & 11 & $21-24$ & $34-43$ & $61-76$ & 97-119 & 169-193 & $257-305$ \\
\hline 9 & 12 & 26 & $45-47$ & $72-81$ & $108-130$ & 209 & $258-327$ \\
\hline 10 & 13 & $27-28$ & $42-50$ & $81-87$ & & 225 & 289-349 \\
\hline 11 & 14 & $26-30$ & $48-54$ & $80-92$ & $113-152$ & $201-241$ & \\
\hline 12 & $14-15$ & 29-31 & $49-57$ & $68-97$ & $129-163$ & 257 & $321-393$ \\
\hline 13 & 15 & 33 & $56-61$ & 97-103 & $129-174$ & $225-270$ & \\
\hline 14 & $15-16$ & $32-35$ & 65 & $97-108$ & $146-185$ & $241-286$ & $353-437$ \\
\hline 15 & 17 & $33-37$ & $56-68$ & $98-113$ & $158-196$ & $258-302$ & $386-459$ \\
\hline 16 & $17-18$ & $36-38$ & $56-71$ & $93-118$ & $147-204$ & & \\
\hline 17 & $17-18$ & 40 & $62-74$ & $112-124$ & $154-212$ & & \\
\hline 18 & $18-19$ & $41-42$ & $65-77$ & $113-129$ & $161-220$ & $281-350$ & \\
\hline 19 & 20 & $37-43$ & $60-80$ & $121-134$ & $172-228$ & & \\
\hline 20 & $19-21$ & $37-45$ & $68-83$ & $121-140$ & $177-236$ & $297-382$ & \\
\hline 21 & 21 & $41-47$ & $72-86$ & $129-145$ & $185-244$ & & \\
\hline 22 & $21-22$ & $41-48$ & $74-89$ & $129-150$ & & $321-414$ & \\
\hline 23 & $22-23$ & $41-50$ & $68-92$ & $126-155$ & & & \\
\hline 24 & $21-23$ & $49-52$ & $81-95$ & 129-161 & & $337-446$ & $513-657$ \\
\hline 25 & 24 & $51-53$ & $84-97$ & $144-166$ & & & \\
\hline 26 & $24-25$ & 55 & $82-100$ & $150-171$ & & $385-478$ & \\
\hline 27 & $22-25$ & $49-56$ & $96-103$ & $145-176$ & $209-290$ & $401-494$ & \\
\hline 28 & $25-26$ & $51-58$ & 97-106 & $145-181$ & $257-298$ & 513 & $577-745$ \\
\hline 29 & $25-27$ & $52-60$ & 97-109 & $161-187$ & $227-306$ & & \\
\hline 30 & $25-27$ & $53-61$ & $96-112$ & $162-192$ & $273-313$ & $401-536$ & 609-789 \\
\hline 31 & $27-28$ & $60-63$ & $89-115$ & $165-197$ & & $386-547$ & $578-811$ \\
\hline 32 & $26-29$ & $57-65$ & $90-118$ & & & & \\
\hline 33 & $28-29$ & $65-66$ & $92-121$ & $193-207$ & & & \\
\hline 34 & $27-30$ & $57-68$ & $98-124$ & $156-213$ & & & \\
\hline 35 & 29-31 & $64-69$ & $112-127$ & & $253-352$ & & \\
\hline 36 & $30-31$ & $64-71$ & $107-130$ & $185-223$ & & & \\
\hline 37 & 29-32 & $66-72$ & $121-132$ & $208-228$ & & & \\
\hline 38 & $28-33$ & $64-74$ & $129-135$ & $193-233$ & $289-375$ & $449-627$ & \\
\hline 39 & 33 & $65-75$ & $120-138$ & $194-239$ & & & \\
\hline 40 & $32-34$ & $75-77$ & $103-141$ & $197-244$ & $293-390$ & 489-560 & \\
\hline 41 & $33-35$ & $65-78$ & $118-144$ & $216-249$ & $308-398$ & & \\
\hline 42 & $33-35$ & $68-80$ & $129-147$ & 209-254 & $307-405$ & $513-672$ & \\
\hline 43 & $33-36$ & $72-81$ & $116-150$ & $226-259$ & $306-413$ & & \\
\hline 44 & $33-37$ & $68-83$ & $130-153$ & $226-264$ & $325-420$ & & \\
\hline 45 & $33-37$ & $80-84$ & $144-156$ & $242-268$ & $304-428$ & & \\
\hline 46 & $34-38$ & $81-86$ & $129-158$ & $243-273$ & & & \\
\hline 47 & $36-38$ & $73-87$ & $120-161$ & & & & \\
\hline 48 & $34-39$ & $77-89$ & $126-164$ & & & & \\
\hline 49 & $36-40$ & $81-90$ & $130-167$ & & & & \\
\hline 50 & 40 & $91-92$ & $130-170$ & $225-291$ & & $561-762$ & \\
\hline
\end{tabular}


TABLE $\mathrm{p}=3$

\begin{tabular}{|c|c|c|c|c|}
\hline$g \backslash q$ & 3 & 9 & 27 & 81 \\
\hline 1 & 7 & 16 & 38 & 100 \\
\hline 2 & 8 & 20 & 48 & 118 \\
\hline 3 & 10 & 28 & 56 & 136 \\
\hline 4 & 12 & 30 & $64-66$ & 154 \\
\hline 5 & $12-13$ & $32-35$ & $69-76$ & $156-172$ \\
\hline 6 & $14-15$ & $35-40$ & $76-86$ & 190 \\
\hline 7 & 16 & $40-43$ & $76-96$ & $160-208$ \\
\hline 8 & $15-18$ & $38-47$ & $92-106$ & 226 \\
\hline 9 & 19 & $48-51$ & $88-116$ & 244 \\
\hline 10 & $19-21$ & $54-55$ & $91-126$ & $226-262$ \\
\hline 11 & $20-22$ & $55-59$ & & \\
\hline 12 & $22-24$ & $55-63$ & 109-146 & 298 \\
\hline 13 & $24-25$ & $60-66$ & $136-156$ & $224-316$ \\
\hline 14 & $24-26$ & $56-70$ & & \\
\hline 15 & 28 & $64-74$ & $136-171$ & $292-352$ \\
\hline 16 & $27-29$ & $74-78$ & $136-178$ & 370 \\
\hline 17 & $24-30$ & $64-82$ & & \\
\hline 18 & $26-31$ & $67-85$ & & \\
\hline 19 & $28-32$ & $84-88$ & & \\
\hline 20 & $30-34$ & $68-91$ & & \\
\hline 21 & $32-35$ & $88-95$ & $163-214$ & $352-458$ \\
\hline 22 & $30-36$ & 78-98 & & \\
\hline 23 & $30-37$ & $92-101$ & & \\
\hline 24 & $31-38$ & 91-104 & $190-235$ & \\
\hline 25 & $36-40$ & $82-108$ & $196-242$ & \\
\hline 26 & $36-41$ & $110-111$ & & \\
\hline 27 & $39-42$ & $91-114$ & & \\
\hline 28 & $37-43$ & $105-117$ & & \\
\hline 29 & $42-44$ & $104-120$ & & \\
\hline 30 & $37-46$ & $91-123$ & & \\
\hline 31 & $40-47$ & $101-127$ & & $460-638$ \\
\hline 32 & $38-48$ & $92-130$ & & \\
\hline 33 & $46-49$ & 109-133 & $220-238$ & \\
\hline 34 & $44-50$ & $111-136$ & & \\
\hline 35 & $47-51$ & $101-139$ & & \\
\hline 36 & $46-52$ & $118-142$ & $244-319$ & 730 \\
\hline 37 & $48-54$ & $120-145$ & & \\
\hline 38 & & 105-149 & & \\
\hline 39 & $46-56$ & $119-152$ & $271-340$ & \\
\hline 40 & $54-57$ & $110-155$ & $244-346$ & \\
\hline 41 & $50-58$ & $119-158$ & & \\
\hline 42 & $49-59$ & $118-161$ & $280-360$ & \\
\hline 43 & $55-60$ & $120-164$ & & \\
\hline 44 & & $119-167$ & & \\
\hline 45 & $49-62$ & $128-170$ & & \\
\hline 46 & $55-63$ & $162-173$ & & \\
\hline 47 & $54-65$ & $154-177$ & 299-395 & \\
\hline 48 & $55-66$ & $163-180$ & $325-402$ & $676-885$ \\
\hline 49 & $63-67$ & $168-183$ & & \\
\hline 50 & $56-68$ & $182-186$ & 299-416 & \\
\hline
\end{tabular}


$q=2:$ sources

\begin{tabular}{|c|c|c|c|}
\hline genus & $\mathrm{N}$ & type & source \\
\hline 1 & 5 & $\overline{V-1}$ & $\overline{\mathrm{S} 1,4}$ \\
\hline 2 & 6 & V-1 & $\mathrm{S} 1,4$ \\
\hline 3 & 7 & $\mathrm{~V}-2$ & $\mathrm{D}$ \\
\hline 4 & 8 & $\mathrm{~V}-2$ & $\mathrm{~S} 1,4$ \\
\hline 5 & 9 & I & $\mathrm{S} 1,4$ \\
\hline 6 & 10 & I & $\mathrm{S} 1,4$ \\
\hline 7 & 10 & I & $\mathrm{S} 1,4$ \\
\hline 8 & 11 & I & $\mathrm{S} 1,4$ \\
\hline 9 & 12 & I & $\mathrm{S} 1,4$ \\
\hline 10 & 13 & I & S5 \\
\hline 11 & 14 & I & S5 \\
\hline 12 & $14-15$ & I & $\mathrm{S} 2,4$ \\
\hline 13 & 15 & I & S5 \\
\hline 14 & $15-16$ & I & $\mathrm{S} 2,4$ \\
\hline 15 & 17 & I & $\mathrm{S} 1,4$ \\
\hline 16 & $17-18$ & I & A \\
\hline 17 & $17-18$ & I & $\mathrm{S} 2,4$ \\
\hline 18 & $18-19$ & I & $\mathrm{S} 2,4$ \\
\hline 19 & 20 & I & $\mathrm{S} 1,4$ \\
\hline 20 & $19-21$ & I & $\mathrm{S} 2,4$ \\
\hline 21 & 21 & $\mathrm{I}$ & $\overline{\mathrm{S} 1,4}$ \\
\hline 22 & $21-22$ & I & Sch \\
\hline 23 & $22-23$ & I & $\mathrm{X}-\mathrm{N}$ \\
\hline 24 & $21-23$ & III & G-V5 \\
\hline 25 & 24 & I & $\mathrm{X}-\mathrm{N}$ \\
\hline 26 & $24-25$ & I & G-V4 \\
\hline 27 & $22-25$ & I & G-V4 \\
\hline 28 & $25-26$ & I & A \\
\hline 29 & $25-27$ & II & $\mathrm{X}-\mathrm{N}$ \\
\hline 30 & $25-27$ & I & A \\
\hline 31 & $27-28$ & II & $\mathrm{X}-\mathrm{N}$ \\
\hline 32 & $26-29$ & I & G-V4 \\
\hline 33 & $28-29$ & I & G-V4 \\
\hline 34 & $27-30$ & II & $\mathrm{X}-\mathrm{N}$ \\
\hline 35 & $29-31$ & I & A \\
\hline 36 & $30-31$ & II & $\mathrm{X}-\mathrm{N}$ \\
\hline 37 & $29-32$ & I & A \\
\hline 38 & $28-33$ & I & G-V4 \\
\hline 39 & 33 & I & $\mathrm{S} 1,4$ \\
\hline 40 & $32-34$ & I & G-V4 \\
\hline 41 & $33-35$ & $\mathrm{I}$ & $\overline{\mathrm{A}}$ \\
\hline 42 & $33-35$ & I & A \\
\hline 43 & $33-36$ & II & X-N \\
\hline 44 & $33-37$ & I & A \\
\hline 45 & $33-37$ & III & G-V5 \\
\hline 46 & $34-38$ & I & G-V4 \\
\hline 47 & $36-38$ & I & G-V4 \\
\hline 48 & $34-39$ & I & G-V4 \\
\hline 49 & $36-40$ & II & $\mathrm{X}-\mathrm{N}$ \\
\hline 50 & 40 & I & $\mathrm{S} 1,4$ \\
\hline
\end{tabular}

$q=4:$ sources

\begin{tabular}{|r|r|r|r|}
\hline genus & N & type & source \\
\hline 1 & 9 & V-1 & S2,4 \\
2 & 10 & V-1 & S2,4 \\
3 & 14 & V-2 & S2,4 \\
4 & 15 & IV & S3 \\
5 & $17-18$ & III & St2 \\
6 & 20 & I & G-V4 \\
7 & $21-22$ & II & N-X3 \\
8 & $21-24$ & I & N-X3 \\
9 & 26 & II & N-X4 \\
10 & $27-28$ & V-3 & G-V4 \\
\hline 11 & $26-30$ & III & G-V5 \\
12 & $29-31$ & I & A \\
13 & 33 & III & St2 \\
14 & $32-35$ & III & G-V5 \\
15 & $33-37$ & II & N-X3 \\
16 & $36-38$ & I & G-V4 \\
17 & 40 & II & N-X4 \\
18 & $41-42$ & II & N-X7 \\
19 & $37-43$ & I & A \\
20 & $37-45$ & I & A \\
\hline 21 & $41-47$ & II & N-X4 \\
22 & $41-48$ & I & A \\
23 & $41-50$ & I & A \\
24 & $49-52$ & III & Sh \\
25 & $51-53$ & II & N-X4 \\
26 & 55 & V-3 & G-V4 \\
27 & $49-56$ & III & G-V4 \\
28 & $51-58$ & I & A \\
29 & $52-60$ & III & Sh \\
30 & $53-61$ & II & N-X7 \\
\hline 31 & $60-63$ & II & N-X4 \\
32 & $57-65$ & I & A \\
33 & $65-66$ & I & L1 \\
34 & $57-68$ & III & G-V4 \\
35 & $64-69$ & III & Sh \\
36 & $64-71$ & II & N-X4 \\
37 & $66-72$ & II & N-X4 \\
38 & $64-74$ & III & Sh \\
39 & $65-75$ & III & G-V7 \\
40 & $75-77$ & II & N-X4 \\
\hline 41 & $65-78$ & III & G-V4 \\
42 & $68-80$ & III & Sh \\
43 & $72-81$ & II & N-X4 \\
44 & $68-83$ & I & G-V4 \\
45 & $80-84$ & I & G-V4 \\
46 & $81-86$ & II & N-X7 \\
47 & $73-87$ & I & A \\
\hline $8-99$ & II & N-X4 \\
\hline & II & N-X4 \\
\hline V-3 & G-V4 \\
\hline & & \\
\hline 19 & &
\end{tabular}


$q=8:$ sources

\begin{tabular}{|c|c|c|c|}
\hline genus & $\mathrm{N}$ & type & source \\
\hline 1 & 14 & $\overline{V-1}$ & $\mathrm{~S} 2,4$ \\
\hline 2 & 18 & V-1 & $\mathrm{S} 2,4$ \\
\hline 3 & 24 & $\mathrm{~V}-2$ & $\mathrm{~S} 2,4$ \\
\hline 4 & $25-27$ & III & G-V5 \\
\hline 5 & $29-32$ & III & G-V4 \\
\hline 6 & $33-35$ & III & St2 \\
\hline 7 & $33-39$ & III & G-V1 \\
\hline 8 & $34-43$ & III & Sh \\
\hline 9 & $45-47$ & II & N-X7 \\
\hline 10 & $42-50$ & III & Sh \\
\hline 11 & $48-54$ & III & G-V5 \\
\hline 12 & $49-57$ & III & G-V5 \\
\hline 13 & 56-61 & III & Sh \\
\hline 14 & 65 & V-4 & $\mathrm{H}-\mathrm{S}$ \\
\hline 15 & $56-68$ & III & Sh \\
\hline 16 & $56-71$ & I & G-V4 \\
\hline 17 & $62-74$ & III & Sh \\
\hline 18 & $65-77$ & III & G-V5 \\
\hline 19 & $60-80$ & III & Sh \\
\hline 20 & $68-83$ & II & N-X6 \\
\hline 21 & $72-86$ & III & G-V5 \\
\hline 22 & $74-89$ & III & $\mathrm{Sh}$ \\
\hline 23 & $68-92$ & I & G-V4 \\
\hline 24 & $81-95$ & III & $\mathrm{Sh}$ \\
\hline 25 & $84-97$ & III & Sh \\
\hline 26 & $82-100$ & III & $\mathrm{Sh}$ \\
\hline 27 & $96-103$ & III & Sh \\
\hline 28 & $97-106$ & III & G-V5 \\
\hline 29 & 97-109 & III & G-V4 \\
\hline 30 & $96-112$ & III & Sh \\
\hline 31 & 89-115 & III & Sh \\
\hline 32 & $90-118$ & III & $\mathrm{Sh}$ \\
\hline 33 & $92-121$ & II & N-X6 \\
\hline 34 & $98-124$ & III & Sh \\
\hline 35 & $112-127$ & III & $\mathrm{Sh}$ \\
\hline 36 & $107-130$ & III & Sh \\
\hline 37 & $121-132$ & III & G-V5 \\
\hline 38 & $129-135$ & III & G-V5 \\
\hline 39 & $120-138$ & III & $\mathrm{Sh}$ \\
\hline 40 & $103-141$ & III & Sh \\
\hline 41 & $118-144$ & III & $\mathrm{Sh}$ \\
\hline 42 & $129-147$ & III & G-V5 \\
\hline 43 & $116-150$ & III & Sh \\
\hline 44 & $130-153$ & III & Sh \\
\hline 45 & $144-156$ & I & G-V4 \\
\hline 46 & $129-158$ & III & G-V4 \\
\hline 47 & $120-161$ & II & N-X6 \\
\hline 48 & $126-164$ & II & N-X6 \\
\hline 49 & $130-167$ & II & N-X6 \\
\hline 50 & $130-170$ & II & N-X6 \\
\hline
\end{tabular}

$q=16:$ sources

\begin{tabular}{|c|c|c|c|}
\hline genus & $\mathrm{N}$ & type & source \\
\hline 1 & 25 & V-1 & $\mathrm{S} 2,4$ \\
\hline 2 & 33 & V-1 & $\mathrm{S} 2,4$ \\
\hline 3 & 38 & $\mathrm{~V}-2$ & $\mathrm{~S} 3,4$ \\
\hline 4 & $45-46$ & V-2 & M-Z-Z \\
\hline 5 & 49-54 & III & G-V4 \\
\hline 6 & 65 & $\mathrm{~V}-2$ & Seg \\
\hline 7 & $63-70$ & II & N-X6 \\
\hline 8 & $61-76$ & III & G-V4 \\
\hline 9 & $72-81$ & II & N-X6 \\
\hline 10 & $81-87$ & II & N-X6 \\
\hline 11 & $80-92$ & II & N-X6 \\
\hline 12 & $68-97$ & III & G-V5 \\
\hline 13 & $97-103$ & III & G-V4 \\
\hline 14 & $97-108$ & III & G-V4 \\
\hline 15 & $98-113$ & III & G-V1 \\
\hline 16 & $93-118$ & III & G-V4 \\
\hline 17 & $112-124$ & III & G-V5 \\
\hline 18 & $113-129$ & III & G-V5 \\
\hline 19 & $121-134$ & II & N-X6 \\
\hline 20 & $121-140$ & III & G-V4 \\
\hline 21 & $129-145$ & III & G-V5 \\
\hline 22 & $129-150$ & III & St2 \\
\hline 23 & $126-155$ & II & N-X6 \\
\hline 24 & $129-161$ & III & G-V5 \\
\hline 25 & $4-166$ & II & N-X6 \\
\hline 26 & $150-171$ & II & N-X6 \\
\hline 27 & $145-176$ & I & A \\
\hline 28 & $145-181$ & III & $\mathrm{Sh}$ \\
\hline 29 & $161-187$ & III & Sh \\
\hline 30 & $162-192$ & III & Do \\
\hline 31 & $165-197$ & $\overline{\mathrm{V}-2}$ & $\overline{G-S}$ \\
\hline 32 & & & \\
\hline 33 & 207 & I & A \\
\hline 34 & $156-213$ & II & N-X6 \\
\hline 35 & & & \\
\hline 36 & -223 & II & N-X7 \\
\hline 37 & $208-228$ & II & N-X7 \\
\hline 38 & $193-233$ & I & A \\
\hline 39 & $194-239$ & III & $\mathrm{Sh}$ \\
\hline 40 & $197-244$ & III & $\mathrm{Sh}$ \\
\hline 41 & $216-249$ & III & $\overline{\mathrm{Sh}}$ \\
\hline 42 & $209-254$ & I & A \\
\hline 43 & $226-259$ & II & N-X7 \\
\hline 44 & $226-264$ & III & $\mathrm{Sh}$ \\
\hline 45 & $242-268$ & III & G-V5 \\
\hline 46 & $243-273$ & II & N-X6 \\
\hline 47 & & & \\
\hline 48 & & & \\
\hline 49 & & & \\
\hline 50 & 225-291 & I & $\mathrm{A}$ \\
\hline
\end{tabular}


$q=32:$ sources

\begin{tabular}{|c|c|c|c|}
\hline genus & $\mathrm{N}$ & type & source \\
\hline 1 & $\overline{44}$ & $\mathrm{~V}-1$ & $\mathrm{~S} 2,4$ \\
\hline 2 & 53 & V-1 & $\mathrm{S} 2,4$ \\
\hline 3 & $63-64$ & $\mathrm{~V}-2$ & M-Z-Z \\
\hline 4 & $70-75$ & $\mathrm{~V}-2$ & M-Z-Z \\
\hline 5 & $76-86$ & IV & Sem \\
\hline 6 & $86-97$ & III & Do \\
\hline 7 & $90-108$ & III & Do \\
\hline 8 & $97-119$ & III & Sh \\
\hline 9 & $108-130$ & III & Sh \\
\hline 10 & & & \\
\hline 11 & $113-152$ & I & $\mathrm{A}$ \\
\hline 12 & $129-163$ & III & G-V1 \\
\hline 13 & $129-174$ & I & A \\
\hline 14 & $146-185$ & III & Do \\
\hline 15 & $158-196$ & $\mathrm{~V}-2$ & H-Le B \\
\hline 16 & $147-204$ & III & Sh \\
\hline 17 & $154-212$ & III & Sh \\
\hline 18 & $161-220$ & I & A \\
\hline 19 & $172-228$ & III & $\mathrm{Sh}$ \\
\hline 20 & $177-236$ & III & Sh \\
\hline 21 & $185-244$ & III & $\mathrm{Sh}$ \\
\hline 22 & & & \\
\hline 23 & & & \\
\hline 24 & & & \\
\hline 25 & & & \\
\hline 26 & & & \\
\hline 27 & $209-290$ & I & A \\
\hline 28 & $257-298$ & III & G-V1 \\
\hline 29 & $227-306$ & III & $\mathrm{Sh}$ \\
\hline 30 & $273-313$ & III & G-V1 \\
\hline 31 & & & \\
\hline 32 & & & \\
\hline 33 & & & \\
\hline 34 & & & \\
\hline 35 & $253-352$ & III & G-V5 \\
\hline 36 & & & \\
\hline 37 & & & \\
\hline 38 & $289-375$ & I & A \\
\hline 39 & & & \\
\hline 40 & $293-390$ & III & $\mathrm{Sh}$ \\
\hline 41 & $308-398$ & III & $\mathrm{Sh}$ \\
\hline 42 & $307-405$ & III & $\mathrm{Sh}$ \\
\hline 43 & $306-413$ & III & Sh \\
\hline 44 & $325-420$ & III & $\mathrm{Sh}$ \\
\hline 45 & $304-428$ & III & Sh \\
\hline 46 & & & \\
\hline 47 & & & \\
\hline 48 & & & \\
\hline 49 & & & \\
\hline 50 & & & \\
\hline
\end{tabular}

$q=64:$ sources

\begin{tabular}{|c|c|c|c|}
\hline genus & $\bar{N}$ & type & source \\
\hline 1 & 81 & $\mathrm{~V}-1$ & $\mathrm{~S} 2,4$ \\
\hline 2 & 97 & $\mathrm{~V}-1$ & $\mathrm{~S} 2,4$ \\
\hline 3 & 113 & $\mathrm{~V}-2$ & $\mathrm{Wi}$ \\
\hline 4 & 129 & $\mathrm{~V}-2$ & Wo \\
\hline 5 & $130-145$ & V-2 & M-Z-Z \\
\hline 6 & 161 & III & G-V3 \\
\hline 7 & 177 & $\mathrm{~V}-2$ & Wo \\
\hline 8 & 169-193 & I & A \\
\hline 9 & 209 & $\mathrm{~V}-5$ & G-S-X \\
\hline 10 & 225 & V-5 & $\mathrm{E}$ \\
\hline 11 & $201-241$ & III & G-V5 \\
\hline 12 & 257 & $\mathrm{~V}-2$ & Wi \\
\hline 13 & $225-270$ & I & A \\
\hline 14 & $241-286$ & I & A \\
\hline 15 & $258-302$ & III & Do \\
\hline 16 & & & \\
\hline 17 & & & \\
\hline 18 & $281-350$ & I & A \\
\hline 19 & & & \\
\hline 20 & $297-382$ & III & Do \\
\hline 21 & & & \\
\hline 22 & $321-414$ & I & A \\
\hline 23 & & & \\
\hline 24 & $337-446$ & I & A \\
\hline 25 & & & \\
\hline 26 & $385-478$ & I & A \\
\hline 27 & $401-494$ & III & G-V5 \\
\hline 28 & 513 & $\mathrm{~V}-2$ & $\mathrm{H}$ \\
\hline 29 & & & \\
\hline 30 & $401-536$ & III & Do \\
\hline 31 & $386-547$ & III & $\overline{\text { Do }}$ \\
\hline 32 & & & \\
\hline 33 & & & \\
\hline 34 & & & \\
\hline 35 & & & \\
\hline 36 & & & \\
\hline 37 & & & \\
\hline 38 & $449-627$ & I & A \\
\hline 39 & & & \\
\hline 40 & $489-650$ & IV & $\mathrm{O}-\mathrm{S}$ \\
\hline 41 & & & \\
\hline 42 & 513-672 & III & Do \\
\hline 43 & & & \\
\hline 44 & & & \\
\hline 45 & & & \\
\hline 46 & & & \\
\hline 47 & & & \\
\hline 48 & & & \\
\hline 49 & & & \\
\hline 50 & $561-762$ & I & $\mathrm{A}$ \\
\hline
\end{tabular}




$$
q=128: \text { sources }
$$

\begin{tabular}{|r|r|r|r|}
\hline genus & $\mathrm{N}$ & type & source \\
\hline 1 & 150 & $\mathrm{~V}-1$ & $\mathrm{~S} 2,4$ \\
2 & 172 & $\mathrm{~V}-1$ & $\mathrm{~S} 2,4$ \\
3 & $191-195$ & $\mathrm{~V}-2$ & $\mathrm{Su}$ \\
4 & $200-217$ & $\mathrm{~V}-2$ & $\mathrm{Wi}$ \\
5 & $227-239$ & $\mathrm{~V}-2$ & $\mathrm{M}-\mathrm{Z}-\mathrm{Z}$ \\
6 & $225-261$ & $\mathrm{~V}-2$ & $\mathrm{Wi}$ \\
7 & $258-283$ & $\mathrm{III}$ & $\mathrm{Do}$ \\
8 & $257-305$ & $\mathrm{~V}-2$ & $\mathrm{Wi}$ \\
9 & $258-327$ & $\mathrm{III}$ & $\mathrm{Do}$ \\
10 & $289-349$ & $\mathrm{III}$ & $\mathrm{G}-\mathrm{V} 3$ \\
\hline 11 & & & \\
12 & $321-393$ & $\mathrm{III}$ & $\mathrm{G}-\mathrm{V} 1$ \\
13 & & & \\
14 & $353-437$ & $\mathrm{III}$ & $\mathrm{G}-\mathrm{V} 3$ \\
15 & $386-459$ & $\mathrm{III}$ & $\mathrm{Do}$ \\
16 & & & \\
17 & & & \\
18 & & & \\
19 & & & \\
20 & & & \\
\hline 21 & & & \\
22 & & & \\
23 & & & \\
24 & $513-657$ & $\mathrm{III}$ & $\mathrm{G}-\mathrm{V} 1$ \\
25 & & & \\
26 & & & \\
27 & & & \\
28 & $577-745$ & $\mathrm{III}$ & $\mathrm{G}-\mathrm{V} 1$ \\
29 & & & \\
30 & $609-789$ & $\mathrm{III}$ & $\mathrm{G}-\mathrm{V} 3$ \\
\hline 31 & $578-811$ & $\mathrm{III}$ & $\mathrm{Do}$ \\
\hline
\end{tabular}


$q=3:$ sources

\begin{tabular}{|r|r|r|r|}
\hline genus & N & type & source \\
\hline 1 & 7 & V-1 & S1,4 \\
2 & 8 & V-1 & S1,4 \\
3 & 10 & V-2 & S2,4 \\
4 & 12 & V-2 & S3 \\
5 & $12-13$ & IV & N-X3 \\
6 & $14-15$ & IV & N-X3 \\
7 & 16 & II & N-X3 \\
8 & $15-18$ & IV & N-X3 \\
9 & 19 & III & G-V4 \\
10 & $19-21$ & III & G-V4 \\
\hline 11 & $20-22$ & I & N-X3 \\
12 & $22-24$ & I & N-X3 \\
13 & $24-25$ & I & N-X3 \\
14 & $24-26$ & IV & N-X3 \\
15 & 28 & III & G-V4 \\
16 & $27-29$ & III & G-V4 \\
17 & $24-30$ & IV & N-X5 \\
18 & $26-31$ & IV & N-X5 \\
19 & $28-32$ & III & G-V5 \\
20 & $30-34$ & III & G-V4 \\
\hline 21 & $32-35$ & IV & N-X5 \\
22 & $30-36$ & III & G-V5 \\
23 & $30-37$ & III & G-V5 \\
24 & $31-38$ & I & A \\
25 & $36-40$ & I & N-X5 \\
26 & $36-41$ & IV & N-X5 \\
27 & $39-42$ & I & N-X5 \\
28 & $37-43$ & IV & N-X5 \\
29 & $42-44$ & I & N-X5 \\
30 & $37-46$ & III & G-V7 \\
\hline 31 & $40-47$ & II & N-X5 \\
32 & $38-48$ & IV & N-X5 \\
33 & $46-49$ & I & A \\
34 & $44-50$ & II & N-X5 \\
35 & $47-51$ & III & G-V7 \\
36 & $46-52$ & III & G-V7 \\
37 & $48-54$ & I & N-X5 \\
38 & & & \\
39 & $46-56$ & III & G-V7 \\
40 & $54-57$ & II & N-X5 \\
\hline 41 & $50-58$ & II & N-X5 \\
42 & $49-59$ & III & G-V7 \\
43 & $55-60$ & II & X-N \\
44 & & & \\
45 & $49-62$ & III & G-V7 \\
46 & $55-63$ & III & G-V4 \\
47 & $54-65$ & I & A \\
48 & $55-66$ & III & G-V4 \\
49 & $63-67$ & III & G-V5 \\
50 & $56-68$ & II & N-X5 \\
\hline & & &
\end{tabular}

$q=9:$ sources

\begin{tabular}{|r|r|r|r|}
\hline genus & N & type & source \\
\hline 1 & 16 & V-1 & S2,4 \\
2 & 20 & V-1 & S2,4 \\
3 & 28 & V-2 & S2,4 \\
4 & 30 & IV & G-V5 \\
5 & $32-35$ & V-3 & G-V4 \\
6 & $35-40$ & II & N-X7 \\
7 & $40-43$ & IV & O-S \\
8 & $38-47$ & III & G-V2 \\
9 & $48-51$ & IV & O-S \\
10 & $54-55$ & III & G-V5 \\
\hline 11 & $55-59$ & III & G-V2 \\
12 & $55-63$ & III & G-V2 \\
13 & $60-66$ & V-3 & G-V4 \\
14 & $56-70$ & III & G-V5 \\
15 & $64-74$ & III & Sh \\
16 & $74-78$ & III & G-V5 \\
17 & $64-82$ & IV & O-S \\
18 & $67-85$ & III & Sh \\
19 & $84-88$ & II & N-X7 \\
20 & $68-91$ & III & Sh \\
\hline 21 & $88-95$ & IV & O-S \\
22 & $78-98$ & II & N-X7 \\
23 & $92-101$ & II & N-X7 \\
24 & $91-104$ & II & N-X7 \\
25 & $82-108$ & III & Sh \\
26 & $110-111$ & V-3 & G-V4 \\
27 & $91-114$ & III & Sh \\
28 & $105-117$ & II & N-X7 \\
29 & $104-120$ & II & N-X7 \\
30 & $91-123$ & III & Sh \\
\hline 31 & $101-127$ & III & Sh \\
32 & $92-130$ & III & Sh \\
33 & $109-133$ & III & Sh \\
34 & $111-136$ & II & N-X7 \\
35 & $101-139$ & III & Sh \\
36 & $118-142$ & III & Sh \\
37 & $120-145$ & II & N-X7 \\
38 & $105-149$ & II & N-X8 \\
39 & $119-152$ & III & Sh \\
40 & $118-155$ & III & Sh \\
\hline 41 & $119-158$ & III & Sh \\
42 & $118-161$ & III & Sh \\
43 & $120-164$ & II & N-X7 \\
44 & $119-167$ & III & Sh \\
45 & $128-170$ & III & Sh \\
46 & $162-173$ & III & Sh \\
47 & $154-177$ & II & N-X7 \\
48 & $163-180$ & III & Sh \\
49 & $168-183$ & II & N-X7 \\
50 & $182-186$ & V-3 & G-V4 \\
\hline & & &
\end{tabular}


$q=27:$ sources

\begin{tabular}{|c|c|c|c|}
\hline genus & $\mathrm{N}$ & type & source \\
\hline 1 & 38 & $\mathrm{~V}-1$ & $\mathrm{~S} 2,4$ \\
\hline 2 & 48 & $\mathrm{~V}-1$ & $\mathrm{~S} 2,4$ \\
\hline 3 & 56 & IV & G-V5 \\
\hline 4 & $64-66$ & III & G-V2 \\
\hline 5 & $68-76$ & IV & Sem \\
\hline 6 & $76-86$ & III & G-V2 \\
\hline 7 & $76-96$ & IV & Sem \\
\hline 8 & $92-106$ & III & G-V5 \\
\hline 9 & $88-116$ & IV & Sem \\
\hline 10 & $91-126$ & I & A \\
\hline 11 & & & \\
\hline 12 & 109-146 & III & G-V2 \\
\hline 13 & $136-156$ & III & G-V2 \\
\hline 14 & & & \\
\hline 15 & $136-171$ & I & A \\
\hline 16 & $136-178$ & I & A \\
\hline 17 & & & \\
\hline 18 & & & \\
\hline 19 & & & \\
\hline 20 & & & \\
\hline 21 & $163-214$ & III & G-V6 \\
\hline 22 & & & \\
\hline 23 & & & \\
\hline 24 & $190-235$ & III & $\mathrm{Sh}$ \\
\hline 25 & $196-242$ & II & N-X7 \\
\hline 26 & & & \\
\hline 27 & & & \\
\hline 28 & & & \\
\hline 29 & & & \\
\hline 30 & & & \\
\hline 31 & & & \\
\hline 32 & & & \\
\hline 33 & $220-298$ & II & N-X7 \\
\hline 34 & & & \\
\hline 35 & & & \\
\hline 36 & 244-319 & III & G-V2 \\
\hline 37 & & & \\
\hline 38 & & & \\
\hline 39 & $271-340$ & III & G-V6 \\
\hline 40 & $244-346$ & III & G-V5 \\
\hline 41 & & & \\
\hline 42 & $280-360$ & II & N-X7 \\
\hline 43 & & & \\
\hline 44 & & & \\
\hline 45 & & & \\
\hline 46 & & & \\
\hline 47 & 299-395 & III & $\mathrm{Sh}$ \\
\hline 48 & $325-402$ & I & A \\
\hline 49 & & & \\
\hline 50 & $299-416$ & III & $\mathrm{Sh}$ \\
\hline
\end{tabular}

$q=81:$ sources

\begin{tabular}{|c|c|c|c|}
\hline genus & $\mathrm{N}$ & type & source \\
\hline 1 & 100 & $\mathrm{~V}-1$ & $\mathrm{~S} 2,4$ \\
\hline 2 & 118 & V-1 & $\mathrm{S} 2,4$ \\
\hline 3 & 136 & $\mathrm{~V}-2$ & Wi \\
\hline 4 & 154 & V-5 & $\mathrm{H}$ \\
\hline 5 & $156-172$ & IV & Sem \\
\hline 6 & 190 & $\mathrm{~V}-2$ & Seg \\
\hline 7 & $160-208$ & $\mathrm{~V}-2$ & Wi \\
\hline 8 & 226 & V-5 & $\mathrm{E}$ \\
\hline 9 & 244 & $\mathrm{~V}-2$ & Wo \\
\hline 10 & $226-262$ & $\mathrm{~V}-2$ & $\mathrm{Wi}$ \\
\hline 11 & & & \\
\hline 12 & 298 & III & G-V2 \\
\hline 13 & $224-316$ & IV & Sem \\
\hline 14 & & & \\
\hline 15 & $292-352$ & IV & $\mathrm{O}-\mathrm{S}$ \\
\hline 16 & 370 & V-5 & $\mathrm{H}$ \\
\hline 17 & & & \\
\hline 18 & & & \\
\hline 19 & & & \\
\hline 20 & & & \\
\hline 21 & $352-458$ & $\mathrm{I}$ & $\bar{A}$ \\
\hline 22 & & & \\
\hline 23 & & & \\
\hline 24 & & & \\
\hline 25 & & & \\
\hline 26 & & & \\
\hline 27 & & & \\
\hline 28 & & & \\
\hline 29 & & & \\
\hline 30 & & & \\
\hline 31 & $460-638$ & I & $\bar{A}$ \\
\hline 32 & & & \\
\hline 33 & & & \\
\hline 34 & & & \\
\hline 35 & & & \\
\hline 36 & 730 & $\mathrm{~V}-2$ & St1 \\
\hline 37 & & & \\
\hline 38 & & & \\
\hline 39 & & & \\
\hline 40 & & & \\
\hline 41 & & & \\
\hline 42 & & & \\
\hline 43 & & & \\
\hline 44 & & & \\
\hline 45 & & & \\
\hline 46 & & & \\
\hline 47 & & & \\
\hline 48 & $676-885$ & I & A \\
\hline 49 & & & \\
\hline 50 & & & \\
\hline
\end{tabular}




\section{ACKNOWLEDGMENTS}

We would like to thank R. Auer, A. Brouwer, N. Elkies, K. Lauter, H. Niederreiter, R. Schoof, S. Sémirat, J.-P. Serre, V. Shabat, H. Stichtenoth, M. Suzuki and C. P. Xing for communicating results to us.

\section{REFERENCES}

[A] R. Auer: Ray class fields of global function fields with many rational places. Report University of Oldenburg, 1998.

[D] L.E. Dickson: Geometrical and invariantive theory of quartic curves modulo 2. Am. J. Math. 37 (1915), 337-354

[Do] J. Doumen: Master's thesis. Leiden University, 1998.

[E] N. Elkies: Private communication, 1997.

[F-T] R. Fuhrmann, F. Torres: The genus of curves over finite fields with many rational points. Manuscripta Math. 89 (1996), 103-106. MR 96m:11046

[G-S] A. Garcia, H. Stichtenoth: A class of polynomials over finite fields. Preprint, 1998.

[G-S-X] A. Garcia, H. Stichtenoth, C.P. Xing: On subfields of the Hermitian function field. Preprint 1998.

[G-V1] G. van der Geer, M. van der Vlugt: Curves over finite fields of characteristic 2 with many rational points. C.R. Acad. Sci. Paris 317, Série I (1993), 593-597. MR 94k:11068

[G-V2] G. van der Geer, M. van der Vlugt: Generalized Hamming weights of codes and curves over finite fields with many points. In: Israel Math Conf. Proc. 9 (1996), 417-432. MR 96m: 11047

[G-V3] G. van der Geer, M. van der Vlugt: Quadratic forms, generalized Hamming weights of codes and curves with many points. J. of Number Theory 59 (1996), 20-36. MR 97i:11068

[G-V4] G. van der Geer, M. van der Vlugt: How to construct curves over finite fields with many points. In: Arithmetic Geometry, (Cortona 1994), F. Catanese Ed., Sympos. Math. 37 Cambridge Univ. Press, Cambridge, 1997, 169-189. MR 98h:11077

[G-V5] G. van der Geer, M. van der Vlugt: Tables for the function $N_{q}(g)$. Regularly updated tables at: http://www.wins.uva.nl/ geer .

[G-V6] G. van der Geer, M. van der Vlugt: Generalized Reed-Muller codes and curves with many points. J. of Number Theory 72 (1998) 257-268. CMP 99:04

[G-V7] G. van der Geer, M. van der Vlugt: Constructing curves over finite fields with many rational points by solving linear equations. Report W 97-29, Leiden University 1997.

[G] V.D. Goppa : Codes on algebraic curves. Sov. Math. Dokl. 24 (1981), 170-172. MR 82k:94017

[H] J.P. Hansen: Group codes and algebraic curves. Mathematica Gottingensis, Schriftenreihe SFB Geometrie und Analysis, Heft 9, 1987.

[H-Le B] G. Haché, D. Le Brigand: Effective construction of algebraic geometry codes. IEEE Trans. Inform. Theory 41 (1995), 1615-1628. MR 97g:94037

[H-S] J.P. Hansen, H. Stichtenoth: Group codes on certain algebraic curves with many rational points. Appl. Algebra Engrg. Comm. Comput. 1 (1990), 67-77. MR 96e:94023

[I] Y. Ihara: Some remarks on the number of rational points of algebraic curves over finite fields. J. Fac. Sci. Tokyo 28 (1981), 721-724. MR 84c:14016

[L1] K. Lauter: Ray class field constructions of curves over finite fields with many rational points. In: Algorithmic Number Theory (Talence 1996), H. Cohen Ed., Lecture Notes in Computer Science 1122, Springer, Berlin, 1996, 187-195. MR 98a:11076

[L2] K. Lauter: Non-existence of a curve over $\mathbb{F}_{3}$ of genus 5 with 14 rational points. Preprint 1998.

[L3] K. Lauter: Improved upper bounds for the number of rational points on algebraic curves over finite fields. Preprint, University of Michigan, 1999

[M-Z-Z] O. Moreno, D. Zinoviev, V. Zinoviev: On several new projective curves over $\mathbb{F}_{2}$ of genus 3,4 and 5. IEEE Trans. Inform. Theory 41 (1995), 1643-1645. MR 97b:14032

[N-X1] H. Niederreiter, C. P. Xing: Quasi-random points and global function fields. In: Finite Fields and Applications, S.D. Cohen, H. Niederreiter Eds., Cambridge Univ. Press, Cambridge 1996, 269-296. MR 97j:11037

[N-X2] H. Niederreiter, C. P. Xing: Explicit global function fields over the binary field with many rational places. Acta Arithm. 75 (1996), 383-396. MR 97d:11177 
[N-X3] H. Niederreiter, C. P. Xing: Cyclotomic function fields, Hilbert class fields and global function fields with many rational places. Acta Arithm. 79 (1997), 59-76. MR 97m:11141

[N-X4] H. Niederreiter, C. P. Xing: Drinfeld modules of rank 1 and algebraic curves with many rational points II. Acta Arithm. 81 (1997), 81-100. CMP 97:14

[N-X5] H. Niederreiter, C. P. Xing: Global function fields fields with many rational points over the ternary field. Acta Arithm. 83 (1998), 65-86. MR 98j:11110

[N-X6] H. Niederreiter, C. P. Xing: Algebraic curves with many rational points over finite fields of characteristic 2. To appear in: Proc. Number Theory Conference (Zakopane 1997), de Gruyter, Berlin.

[N-X7] H. Niederreiter, C. P. Xing: A general method of constructing global function fields with many rational places. To appear in: Algorithmic Number Theory (Portland 1998), Lecture Notes in Comp. Science, Springer, Berlin.

[N-X8] H. Niederreiter, C. P. Xing: Nets, $(t, s)$-sequences and algebraic geometry. To appear in Pseudo- and quasi-random point sets, P. Hellekalek, G. Larcher, Eds. Lecture Notes in Statistics, Springer, New York, 1998.

[O-S] F. Özbudak, H. Stichtenoth: Curves with many points and configurations of hyperplanes over finite fields. Preprint 1998.

[Sch] R. Schoof: Algebraic curves and coding theory. UTM 336, Univ. of Trento, 1990.

[Seg] B. Segre: Introduction to Galois geometries. Atti Acad. Naz. Lincei (Mem. Cl. Sci. Fis. Mat. Natur.) 8 (1967), 133-236. MR 39:206

[Sem] S. Sémirat: Genus theory for quadratic fields and applications. Preprint Université Paris VI, 1998.

[S1] J-P. Serre: Sur le nombre de points rationnels d'une courbe algébrique sur un corps fini. C.R. Acad. Sci. Paris 296, Série I (1983), 397-402. (= Oeuvres III, No. 128, 658-663). MR 85b:14027; MR 89h:01109c

[S2] J-P. Serre : Nombre de points des courbes algébriques sur $\mathbb{F}_{q}$. Sém. de Théorie des Nombres de Bordeaux, 1982/83, exp. no. 22. (= Oeuvres III, No. 129, 664-668). MR 86d:11051; MR 89h:01109c

[S3] J-P. Serre : Quel est le nombre maximum de points rationnels que peut avoir une courbe algébrique de genre $g$ sur un corps fini $\mathbb{F}_{q}$ ? Résumé des Cours de 1983-1984. (=Oeuvres III, No. 132, 701-705). MR 89h:01109c

[S4] J-P. Serre: Rational points on curves over finite fields. Notes of lectures at Harvard University 1985.

[S5] J-P. Serre: Letter to G. van der Geer, September 1, 1997.

[Sh] V. Shabat: Unpublished manuscript, University of Amsterdam, 1997/98.

[St1] H. Stichtenoth: Self-dual Goppa codes. J. Pure and Appl. Algebra 55 (1988), 199-211. MR 90a: 11150

[St2] H. Stichtenoth: Algebraic-geometric codes associated to Artin-Schreier extensions of $\mathbb{F}_{q}(z)$. In: Proc. 2nd Int. Workshop on Alg. and Comb. Coding Theory, Leningrad (1990), 203-206.

[S-V] K.O. Stöhr, J. F. Voloch: Weierstrass points and curves over finite fields. Proc. London Math. Soc. 52 (1986), 1-19. MR 87b:14010

[Su] M. Suzuki: Private communication, 1998.

[Wi] M. Wirtz : Konstruktion und Tabellen linearer Codes. Westfälische WilhelmsUniversität Münster, 1991.

[Wo] J. Wolfmann: Nombre de points rationnels de courbes algébriques sur des corps finis associées à des codes cycliques. C.R. Acad. Sci. Paris 305, Série I (1987), 345-348. MR 88k: 11025

[X-N] C. P. Xing, H. Niederreiter: Drinfeld modules of rank 1 and algebraic curves with many rational points. Report Austrian Academy of Sciences, Vienna, 1996.

Faculteit Wins, Universiteit van Amsterdam, Plantage Muidergracht 24, 1018 TV Amsterdam, The Netherlands

E-mail address: geer@wins.uva.nl

Mathematisch Instituut, Rijksuniversiteit te Leiden, Niels Bohrweg 1, 2300 RA LeiDEN, The Netherlands

E-mail address: vlugt@wi.leidenuniv.nl 